\title{
SOME FINITE QUOTIENTS OF THE MAPPING CLASS GROUP OF A SURFACE
}

\author{
PATRICIA L. SIPE
}

\begin{abstract}
Let $S$ be a smooth, oriented, compact surface of genus $p \geq 2$, and $\operatorname{Mod}_{p}$ its Teichmüller modular group (or mapping class group). Let $T_{1}(S)$ denote the unit tangent bundle, and let $n$ be an integer dividing $2 p-2 . \operatorname{Mod}_{p}$ acts on the finite set $\Phi_{n}$, the elements of which are certain homomorphisms from $H_{1}\left(T_{1}(S), \mathbf{Z}_{n}\right)$ to $\mathbf{Z}_{n}$. In previous work of the author, these homomorphisms arose as the topological description of the $n$th roots of the canonical bundle of the universal Teichmüller curve; however, a topological approach is taken here. The subgroups of $G_{p, n}$ which leave all elements of $\Phi_{n}$ fixed are subgroups of finite index in $\operatorname{Mod}_{p}$. Let $Q_{n}=\operatorname{Mod}_{p} / G_{p, n}$. The elements of $Q_{n}$ are characterized algebraically. $Q_{n}$ is an extension of $\left(2 Z_{n}\right)^{2 p}$ by the symplectic group $\operatorname{Sp}\left(p, \mathbf{Z}_{n}\right)$ (and in the case of $n$ odd, $Q_{n}$ is a semidirect product).
\end{abstract}

1. Introduction. Let $S$ be a smooth, oriented, compact surface of genus $p \geq 2$. In $[8,9]$, the author studied an action of the Teichmüller modular group on the $n$th roots of the canonical bundle of the universal Teichmüller curve $(n$ is an integer dividing $2 p-2$ ). Topological descriptions of the $n$th roots and the action were given, and the subgroups $G_{p, n}$ of the modular group $\operatorname{Mod}_{p}$ which fix all $n$th roots were characterized (these results are summarized here in $\S 2$ ). The purpose of this paper is to describe the finite quotients $Q_{n}=\operatorname{Mod}_{p} / G_{p, n}$. The main result (see $\S 2$ for a more precise statement) is

THEOREM. $Q_{n}$ is an extension of $\left(2 \mathbf{Z}_{n}\right)^{2 p}$ by the symplectic group $\operatorname{Sp}\left(p, \mathbf{Z}_{n}\right)$.

Here $\mathbf{Z}_{n}$ denotes the integers modulo $n$, and $2 \mathbf{Z}_{n}$ is the subgroup of that group generated by 2 (so if $n$ is odd, $\left.\mathbf{Z}_{n} \approx 2 \mathbf{Z}_{n}\right)$. $\left(2 \mathbf{Z}_{n}\right)^{2 p}$ is the finite group of $2 p$-vectors with entries in $2 \mathbf{Z}_{n}$.

The results are obtained by analyzing a representation of $\operatorname{Mod}_{p}$ in the set of $(2 p+1) \times(2 p+1)$ matrices with coefficients in $\mathbf{Z}_{n}$ instead of the usual representation in the set of $2 p \times 2 p$ matrices. The subgroup $G_{p, n}$ is the kernel of this representation; the main work of the paper is to characterize the image.

In his preliminary study of $n$th roots [3], Earle proved that if $n=2$, a diffeomorphism class $f \in \operatorname{Mod}_{p}$ is in $G_{p, n}$ if and only if it induces the identity on $H_{1}\left(S, \mathbf{Z}_{2}\right)$. The above theorem reduces to this statement if $n=2$. In this case, the action of $\operatorname{Sp}\left(p, \mathbf{Z}_{2}\right)$ on square roots agrees with the action of $\operatorname{Mod}_{p}$. These square roots are the spin structures studied by Atiyah [1]. Johnson [4] gave a particularly appealing description of the square roots and the action of $\operatorname{Sp}\left(p, \mathbf{Z}_{2}\right)$ which is discussed here in $\S 3$.

Received by the editors January 29, 1985 and, in revised form, July 9, 1985. The contents of this paper were presented at a Special Session "Surfaces and 3-Manifolds" at the AMS meeting in Fairfield, Connecticut, October 28-29, 1983 and at the ICM, Warsaw, August 1983.

1980 Mathematics Subject Classification. Primary 57N05, 20H05; Secondary 30F10, 11F06, $32 \mathrm{G} 15$. 
The case $n>2$ is more complicated. It is no longer true that $f \in G_{p, n}$ if and only if it acts as the identity on $H_{1}\left(S, \mathbf{Z}_{n}\right)$. Earle [3] gave an example of a diffeomorphism which acts trivially on $H_{1}(S, \mathbf{Z})$, yet does not belong to $G_{p, 2 p-2}$. This example was discussed in [9], using geometric methods, and in fact leads to a collection of examples (Lemma 1) which will be important in $\S 4$, which contains the proof of the theorem.

The author wishes to thank Clifford J. Earle for many useful discussions and his continued encouragement. She also thanks Dennis Johnson, John McCarthy and Roger Lyndon for helpful conversations, and the referee for careful comments.

\section{The $n$th roots and the action of the modular group.}

2.1. We begin by summarizing the notation and results of [9]. Fix a canonical basis $\left\{A_{1}, \ldots, A_{p}, B_{1}, \ldots, B_{p}\right\}$ for the first homology group $H_{1}(S, \mathbf{Z})$; that is, fix simple closed curves so that $A_{i} \cdot A_{j}=B_{i} \cdot B_{j}=0, A_{i} \cdot B_{j}=\delta_{i j}$. Here $\cdot$ is the intersection pairing on $H_{1}(S, \mathbf{Z})$. It will be useful to think of $S$ as equipped with a Riemannian metric, with respect to which $S$ is symmetric, so that the curves $\left\{A_{i}, B_{i}\right\}_{i=1}^{p}$ are parameterized by arc-length, and $r_{p}$, the rotation of order $p$, maps $A_{i}$ to $A_{i+1}$ and $B_{i}$ to $B_{i+1}$ unless $i=p$, in which case we have $A_{p} \mapsto A_{1}$ and $B_{p} \mapsto B_{1}$. Let $T_{1}(S)$ denote the unit tangent bundle of $S$, and denote the fiber class in $H_{1}\left(T_{1}(S), \mathbf{Z}\right)$ by $\alpha$. Throughout the paper, $\approx$ denotes group isomorphism.

Proposition 1. The fiber class $\alpha$ in $H_{1}\left(T_{1}(S), \mathbf{Z}\right)$ has order $2 p-2$. If $\left\{\alpha_{1}, \alpha_{2}\right.$, $\left.\ldots, \alpha_{p}, \beta_{1}, \ldots, \beta_{p}\right\}$ is any basis of $H_{1}(S, \mathbf{Z})$, and $\left\{\alpha_{1}^{\prime}, \alpha_{2}^{\prime}, \ldots, \alpha_{p}^{\prime}, \beta_{1}^{\prime}, \ldots, \beta_{p}^{\prime}\right\}$ are any lifts of these elements to paths in $T_{1}(S)$, then the collection $\left\{\alpha_{1}^{\prime}, \ldots, \alpha_{p}^{\prime}, \beta_{1}^{\prime}, \ldots, \beta_{p}^{\prime}\right.$, $\alpha$ forms a basis for $H_{1}\left(T_{1}(S), \mathbf{Z}\right) \approx \mathbf{Z}^{2 p} \oplus \mathbf{Z}_{2 p-2}$.

PROOF. Let $T_{0}(S)$ be the tangent bundle of the surface $S$ with its zero set removed. The natural inclusion $i: T_{1}(S) \rightarrow T_{0}(S)$ is a homotopy equivalence; $T_{1}(S)$ is a deformation retract of $T_{0}(S)$. Therefore, we may identify $H_{1}\left(T_{1}(S), \mathbf{Z}_{n}\right)$ with $H_{1}\left(T_{0}(S), \mathbf{Z}_{n}\right)$ and under this identification, $\alpha \in H_{1}\left(T_{1}(S), \mathbf{Z}_{n}\right)$ corresponds to the loop around the origin in the fiber of $T_{0}(S)$. Keeping this identification in mind, we see that this proposition is a restatement of Proposition $2.5[9, \mathrm{p} .74]$.

COROLLARY. If $n$ is an integer dividing $2 p-2$, then $H_{1}\left(T_{1}(S), \mathbf{Z}_{n}\right) \approx\left(\mathbf{Z}_{n}\right)^{2 p+1}$ with basis $\left\{\alpha_{1}^{\prime}, \ldots, \alpha_{p}^{\prime}, \beta_{1}^{\prime}, \ldots, \beta_{p}^{\prime}, \alpha\right\}$.

REMARK. If $\gamma^{\prime}$ is a lift of a path $\gamma$ in $S$, then the image of $\gamma^{\prime}$ under the natural projection $T_{1}(S) \rightarrow S$ is $\gamma$. If $\gamma^{\prime}$ and $\gamma^{\prime \prime}$ are two lifts of $\gamma$ to $T_{1}(S)$, then $\gamma^{\prime}-\gamma^{\prime \prime}=m \alpha$ for some integer $m$. Note that there is a "natural" way of lifting paths; a path $\gamma$ in $S$ lifts to $\bar{\gamma}$ in $T_{1}(S)$, where

$$
\bar{\gamma}(t)=\left(\gamma(t), \gamma^{\prime}(t)\right) \text {. }
$$

This lifting, however, is not well defined on homology classes. For example, a small loop homotopic to a point lifts to $\alpha$ or $-\alpha$ depending upon its orientation.

DEFINITION. Let $n$ be an integer dividing $2 p-2$.

$$
\Phi_{n}=\left\{\text { homomorphisms } \phi: H_{1}\left(T_{1}(S), \mathbf{Z}_{n}\right) \rightarrow \mathbf{Z}_{n} \text { such that } \phi(\alpha)=-1\right\} \text {. }
$$

The set $\Phi_{n}$ is a finite set of $n^{2 p}$ elements. In [9] this set arises as a topological description of those line bundles which are $n$th roots of the canonical bundle of the universal Teichmüller curve. The reason for the condition $n \mid 2 p-2$ is clearer 


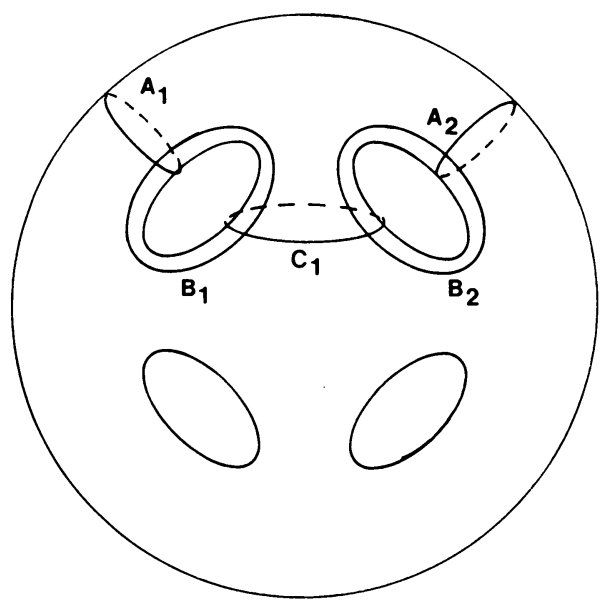

FIGURE 1

in that context; if an $n$th root is to exist, then $n$ must divide the Chern class of the canonical bundle, $2 p-2$. Taking this topological description as the definition perhaps obscures the motivation for studying the sets $\Phi_{n}$, but makes possible a simpler approach to the study of the subgroups of the modular group in which we are interested here.

2.2. Let $\operatorname{Diff}^{+}(S)$ be the group of sense-preserving diffeomorphisms of $S$, and $\operatorname{Diff}_{0}(S)$ the normal subgroup of those homotopic to the identity. The mapping class group (or Teichmüller modular group) is defined by $\operatorname{Mod}_{p}=\operatorname{Diff}^{+}(S) / \operatorname{Diff}_{0}(S)$.

If $\gamma$ is a simple closed curve, let $f_{\gamma}$ denote the Dehn twist about $\gamma$ (here, the same notation is used to denote a diffeomorphism of $S$ and the class in $\operatorname{Mod}_{p}$ it represents). It is well known that $\operatorname{Mod}_{p}$ is generated by Dehn twists [5]. Fix $C_{1}$, a simple closed curve representing $A_{2} A_{1}^{-1}$ (see Figure 1). The four elements $f_{A_{1}}, f_{B_{1}}, f_{C_{1}}$ and $r_{p}$, the rotation of order $p$, form a set of generators for $\operatorname{Mod}_{p}$ $[6,2]$.

A diffeomorphism $f \in \operatorname{Mod}_{p}$ induces a map on homology $\rho(f): H_{1}(S, \mathbf{Z}) \rightarrow$ $H_{1}(S, \mathbf{Z})$. Thus, $\rho$ is a homomorphism $\rho: \operatorname{Mod}_{p} \rightarrow \operatorname{Sp}(p, \mathbf{Z})$ where $\operatorname{Sp}(p, \mathbf{Z})$ denotes the symplectic group of transformations of $H_{1}(S, \mathbf{Z})$. These are represented by $2 p \times 2 p$ matrices $M$ which satisfy $M^{t} J M=J$. Here $J=\left(\begin{array}{cc}0 & -I \\ I & 0\end{array}\right)$ and $M^{t}$ is the transpose of the matrix $M$. The $\operatorname{map} \rho: \operatorname{Mod}_{p} \rightarrow \operatorname{Sp}(p, \mathbf{Z})$ is onto (see, for example $\left[\mathbf{7}\right.$, p. 125]). It follows that the $\operatorname{map} \rho_{n}: \operatorname{Mod}_{p} \rightarrow \operatorname{Sp}\left(p, \mathbf{Z}_{n}\right)$, the group of symplectic matrices with coefficients in $\mathbf{Z}_{n}$, is also onto.

$\operatorname{Mod}_{p}$ acts on $H_{1}\left(T_{1}(S), \mathbf{Z}_{n}\right)$. For the differential $D f$ induces a homomorphism $(D f)_{*}: H_{1}\left(T_{1}(S), \mathbf{Z}_{n}\right) \rightarrow H_{1}\left(T_{1}(S), \mathbf{Z}_{n}\right)$. This gives an action of $\operatorname{Mod}_{p}$ on the set $\Phi_{n}$. If $\phi \in \Phi_{n}$, then $\phi: H_{1}\left(T_{1}(S), \mathbf{Z}_{n}\right) \rightarrow \mathbf{Z}_{n}$ and $f \in \operatorname{Mod}_{p}$ acts by the rule

$$
f \cdot \phi=\phi \circ\left(D f^{-1}\right)_{*} .
$$

The condition $(f \cdot \phi)(\alpha)=-1$ is satisfied because $\left(D f^{-1}\right)_{*}$ takes the fiber class $\alpha$ to itself. By definition, $G_{p, n}$ is the subgroup of $\operatorname{Mod}_{p}$ which fixes all elements of $\Phi_{n}$. 
Since $\Phi_{n}$ is a finite set of $n^{2 p}$ elements, and $G_{p, n}$ is the kernel of the homomorphism $\operatorname{Mod}_{p} \rightarrow \operatorname{Perm}\left(n^{2 p}\right), G_{p, n}$ is a subgroup of finite index in $\operatorname{Mod}_{p}$. The following three theorems were proved in [9], although with the approach taken in this paper, Theorem $\mathrm{A}$ is essentially a definition.

THEOREM A. $f \in G_{p, n}$ if and only if $\left(D f^{-1}\right)_{*}$ is the identity on $H_{1}\left(T_{1}(S), \mathbf{Z}_{n}\right)$.

THEOREM B. The action of $\operatorname{Mod}_{p}$ on $\Phi_{n}$ can be (is) computed for a set of generators for $\operatorname{Mod}_{p}$.

THEOREM C. The image of $G_{p, n}$ under the mapping $\rho_{n}: \operatorname{Mod}_{p} \rightarrow \operatorname{Sp}\left(p, \mathbf{Z}_{n}\right)$ is precisely the congruence subgroup of level $n$.

The Torelli group is by definition the subgroup of $\operatorname{Mod}_{p}$ which acts as the identity on $H_{1}(S, \mathbf{Z})$. Note that the subgroups $G_{p, n}$ neither contain nor are they contained in the Torelli group.

The mapping $\rho_{n}: \operatorname{Mod}_{p} \rightarrow \operatorname{Sp}\left(p, \mathbf{Z}_{n}\right)$ factors through $Q_{n}$ to give a map which we also denote by $\rho_{n}$. The statement of the theorem is

THEOREM. The homomorphism $\rho_{n}: Q_{n} \rightarrow \operatorname{Sp}\left(p, \mathbf{Z}_{n}\right)$ has kernel isomorphic to $\left(2 \mathbf{Z}_{n}\right)^{2 p}$. That is,

$$
0 \rightarrow\left(2 \mathbf{Z}_{n}\right)^{2 p} \rightarrow Q_{n} \stackrel{\rho_{n}}{\rightarrow} \operatorname{Sp}\left(p, \mathbf{Z}_{n}\right) \rightarrow 0
$$

is a short exact sequence.

COROLLARY. If $n$ is odd, the exact sequence splits and $Q_{n}$ is the semidirect product $\left(\mathbf{Z}_{n}\right)^{2 p} \ltimes \mathrm{Sp}\left(p, \mathbf{Z}_{n}\right)$.

2.3. We have fixed a basis for $H_{1}(S, \mathbf{Z})$. If we let $\left\{A_{1}^{\prime}, A_{2}^{\prime}, \ldots, A_{p}^{\prime}, B_{1}^{\prime}, \ldots, B_{p}^{\prime}, \alpha\right\}$ be a basis for $H_{1}\left(T_{1}(S), \mathbf{Z}_{n}\right)$ as in Proposition 1, then the elements of $\Phi_{n}$ can be written as column vectors of length $2 p+1$ with entries in $\mathbf{Z}_{n}$ (the final entry is always -1 , since $\phi(\alpha)=-1)$.

Let $\delta$ be the map from $\operatorname{Mod}_{p}$ to the set of $(2 p+1) \times(2 p+1)$ matrices with entries in $\mathbf{Z}_{n}$ which assigns to each $f \in \operatorname{Mod}_{p}$ the matrix representing the induced map $\left(D f^{-1}\right)_{*}$ with respect to this basis. Then the action of $\operatorname{Mod}_{p}$ is described by right multiplication by $\delta(f)$, and $\delta(f)$ is of the form

$$
\left(\begin{array}{cc}
\rho_{n}\left(f^{-1}\right) & 0 \\
v(f) & 1
\end{array}\right)
$$

where $\rho_{n}\left(f^{-1}\right) \in \operatorname{Sp}\left(p, \mathbf{Z}_{n}\right)$ and $v(f) \in\left(\mathbf{Z}_{n}\right)^{2 p}$. Since the kernel of $\delta$ is $G_{p, n}$, the image of $\operatorname{Mod}_{p}$ under $\delta$ is isomorphic to $Q_{n}$. Identifying $Q_{n}$ with the image allows us to speak of elements of $Q_{n}$ as matrices. Our goal is to identify which matrices are in the image.

3. The case $n=2$. Johnson [4], in the process of giving a topological description of Atiyah's invariant on spin structures, found that there is a natural lifting of mod 2 homology classes on the surface $S$ to mod 2 homology classes on its unit tangent bundle. We will need the definition of this lift for our study of the case $n>2$ in the next section.

Recall that for any simple closed curve $\tau$ in $S$, we have defined a lift $\bar{\tau}$, a path in $T_{1}(S)$. Let $\gamma$ be any homology class in $H_{1}(S, \mathbf{Z})$. Represent it by a collection of simple closed curves $\left\{\gamma_{1}, \ldots, \gamma_{k}\right\}$. Define $\tilde{\gamma} \in H_{1}\left(T_{1}(S), \mathbf{Z}_{2}\right)$ to be the $\bmod 2$ homology class of $\sum_{i=1}^{k} \bar{\gamma}_{i}+k \alpha$. 
THEOREM (JOHNSON [4]). If $\left[\gamma_{1}\right]=\left[\gamma_{2}\right]$ in $H_{1}(S, \mathbf{Z})$, then $\left[\tilde{\gamma}_{1}\right]=\left[\tilde{\gamma}_{2}\right]$ in $H_{1}\left(T_{1}(S), \mathbf{Z}_{2}\right)$. Thus, “ " is a natural lifting from $H_{1}(S, \mathbf{Z})$ to $H_{1}\left(T_{1}(S), \mathbf{Z}_{2}\right)$. This natural lifting is not a homomorphism, rather,

$$
\left(\widetilde{\gamma_{1}+\gamma_{2}}\right)=\tilde{\gamma}_{1}+\tilde{\gamma}_{2}+\left(\gamma_{1} \cdot \gamma_{2}\right) \alpha \text {. }
$$

Johnson goes on to prove that the spin structures considered by Atiyah (which for us are the elements of $\Phi_{2}$ ) can be identified with certain quadratic forms. The quadratic forms he considers are homomorphisms $q: H_{1}\left(S, \mathbf{Z}_{2}\right) \rightarrow \mathbf{Z}_{2}$ such that $q(a+b)=q(a)+q(b)+(a \cdot b)$, that is, the associated bilinear pairing is the intersection pairing. The identification is as follows: With each $\phi \in \Phi_{2}, \phi: H_{1}\left(T_{1}(S), \mathbf{Z}_{2}\right) \rightarrow \mathbf{Z}_{2}$, associate the quadratic form given by the rule $q_{\phi}(a)=\phi(\tilde{a})$. Johnson shows that the Arf invariant of the quadratic form is the Atiyah invariant of the associated spin structure. Two elements of $\Phi_{2}$ are in the same orbit of the action if and only if they have the same Arf invariant.

4. The case $n>2$. We now assume that $n>2$, and because $n \mid 2 p-2$, this also means that $p>2$ for the remainder of the paper. If $n \neq 2$, we cannot hope to define a natural lifting of $H_{1}(S, \mathbf{Z})$ to $H_{1}\left(T_{1}(S), \mathbf{Z}_{n}\right)$. If $\gamma \in H_{1}(S, \mathbf{Z})$ is represented by $\left\{\gamma_{1}, \ldots, \gamma_{k}\right\}$, the $\bmod n$ homology class of $\sum_{i=1}^{k} \bar{\gamma}_{1}+k \alpha$ generally will depend on the choice of simple closed curves representing $\gamma$. Nevertheless, if we start with a particular simple closed curve $\tilde{\tau}$, we can think of $\tilde{\tau}=\bar{\tau}+\alpha$ as a path in $T_{1}(S)$ which therefore represents a homology class in $H_{1}\left(T_{1}(S), \mathbf{Z}_{n}\right)$. So by starting with the particular set of curves $\left\{A_{1}, \ldots, B_{p}\right\}$ described in $\S 1$, we can form the basis $\left\{\tilde{A}_{1}, \ldots, \tilde{B}_{p}, \alpha\right\}$ as in Proposition 1. The matrix representations of elements of $\Phi_{n}$ and of the maps $\left(D f^{-1}\right)_{*}$ can then be written in terms of this basis, and we will assume for the remainder of this paper that all vectors and matrices are so expressed. Note, however, that the calculations in [9] were done with respect to the basis $\left\{\bar{A}_{1}, \ldots, \bar{B}_{p}, \alpha\right\}$ and not with respect to the one described here. The results are easily translated, of course, because of the relations $\tilde{A}_{i}=\bar{A}_{i}+\alpha, \tilde{B}_{i}=\bar{B}_{i}+\alpha$.

Under the map $\delta$, the elements of $Q_{n}$ are identified with matrices of the form $\left[\begin{array}{cc}M & 0 \\ v & 1\end{array}\right]$ which are expressed with respect to the basis $\left\{\tilde{A}_{i}, \tilde{B}_{i}, \alpha\right\}_{i=1}^{p}$ and induced by elements of $\operatorname{Mod}_{p}$. Not all possible matrices of this form lie in the image of $\operatorname{Mod}_{p}$. The following proposition and its corollary characterize which matrices occur in this way, and are essentially restatements of the theorem (stated in \$2) and its corollary.

Proposition. The matrix $\left[\begin{array}{cc}M & 0 \\ v & 1\end{array}\right]$, expressed with respect to $\left\{\tilde{A}_{i}, \tilde{B}_{i}, \alpha\right\}_{i=1}^{p}$ is induced by $f \in \operatorname{Mod}_{p}$ if and only if $M \in \operatorname{Sp}\left(p, \mathbf{Z}_{n}\right)$ and

$$
\operatorname{Diag}\left(M^{t} N M\right)-v \in\left(2 \mathbf{Z}_{n}\right)^{2 p}
$$

where $N$ is the $2 p \times 2 p$ matrix $\left(\begin{array}{ll}0 & I \\ 0 & 0\end{array}\right)$. Here, for any square matrix $M, M^{t}$ is its transpose and $\operatorname{Diag}(M)$ denotes the vector whose ith component is $M_{i i}$.

COROLlaRY. If $n$ is odd, $\operatorname{Mod}_{p} / G_{p, n}$ is the semidirect product of $\left(\mathbf{Z}_{n}\right)^{2 p}$ by $\operatorname{Sp}\left(p, \mathbf{Z}_{n}\right)$.

The remainder of this section is devoted to the proof of the proposition. Notice that the theorem follows easily from the proposition, since $\rho_{n}: Q_{n} \rightarrow \operatorname{Sp}\left(p, \mathbf{Z}_{n}\right)$ takes $\left[\begin{array}{cc}M & 0 \\ v & 1\end{array}\right] \rightarrow M$ and $\operatorname{ker}\left(\rho_{n}\right)$ is the subgroup of matrices of the form $\left[\begin{array}{ll}I & 0 \\ v & 1\end{array}\right]$ where 


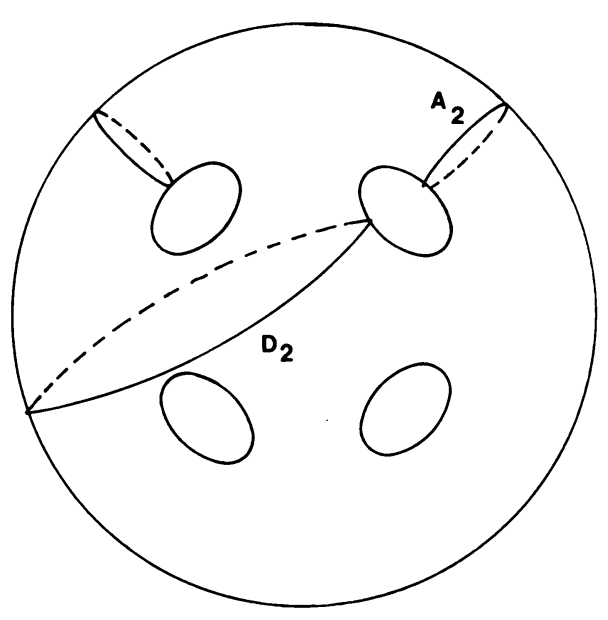

FIGURE 2

$v \in\left(2 \mathbf{Z}_{n}\right)^{2 p}$. Note that by the proposition, the matrices of the form $\left[\begin{array}{ll}I & 0 \\ v & 1\end{array}\right]$ are in the image of the map from $\operatorname{Mod}_{p}$ into $(2 p+1) \times(2 p+1)$ matrices. Those statements are independent of the basis chosen for $H_{1}\left(T_{1}(S), \mathbf{Z}_{n}\right)$. However, formula $(*)$ holds only for the particular basis $\left\{\tilde{A}_{i}, \tilde{B}_{i}, \alpha\right\}_{i=1}^{p}$ described here. One can easily check whether condition (*) is satisfied. For let $M=\left(M_{i j}\right)_{i, j=1}^{2 p}$. Each column $\left(M_{i j}\right)_{i=1}^{2 p}$ of $M$ consists of two vectors of length $p$ whose dot product $\sum_{i=1}^{p}\left(M_{i j}\right)\left(M_{(i+p) j}\right) \in \mathbf{Z}_{n}$. If it agrees, $\bmod 2$ with $v_{j}$ (the $j$ th entry of $v$ ) for every $1 \leq j \leq 2 p$, then $(*)$ holds.

The following lemmas are used in the proof of the proposition. We begin by constructing examples of elements in $\operatorname{Mod}_{p}$ which act trivially on $H_{1}\left(S, \mathbf{Z}_{n}\right)$ yet do not act trivially on $\Phi_{n}$.

LEMMA 1. Given any vector $v=\left(v_{1}, v_{2}\right) \in\left(2 \mathbf{Z}_{n}\right)^{p} \times\left(2 \mathbf{Z}_{n}\right)^{p}=\left(2 \mathbf{Z}_{n}\right)^{2 p}$ there is a diffeomorphism which acts trivially on $H_{1}(S, \mathbf{Z})$ and acts on $H_{1}\left(T_{1}(S), \mathbf{Z}_{n}\right)$ by

$$
\left(\begin{array}{ccc}
I & 0 & 0 \\
0 & I & 0 \\
v_{1} & v_{2} & 1
\end{array}\right)
$$

(here $I$ is the $p \times p$ identity matrix).

PROOF. Fix a simple closed curve $D_{2}$ representing $A_{1} B_{1} A_{1}^{-1} B_{1}^{-1} A_{2}^{-1}$ as in Figure 2. Let $g \in \operatorname{Mod}_{p}$ be given by $f_{A_{2}}^{-1} \circ f_{D_{2}}$.

$$
\left(D g^{-1}\right)_{*}=\left(\begin{array}{ccc}
I & 0 & 0 \\
0 & I & 0 \\
0 & w & 1
\end{array}\right),
$$

where $w=\left(w_{1}, w_{2}, \ldots, w_{p}\right)$ has $w_{2}=2$, all other entries zero (see $\left.[\mathbf{9}, \S 4]\right)$. The rotation diffeomorphism $r_{p}$ is represented by the matrix

$$
\left(\begin{array}{ccc}
R & 0 & 0 \\
0 & R & 0 \\
0 & 0 & 1
\end{array}\right)
$$




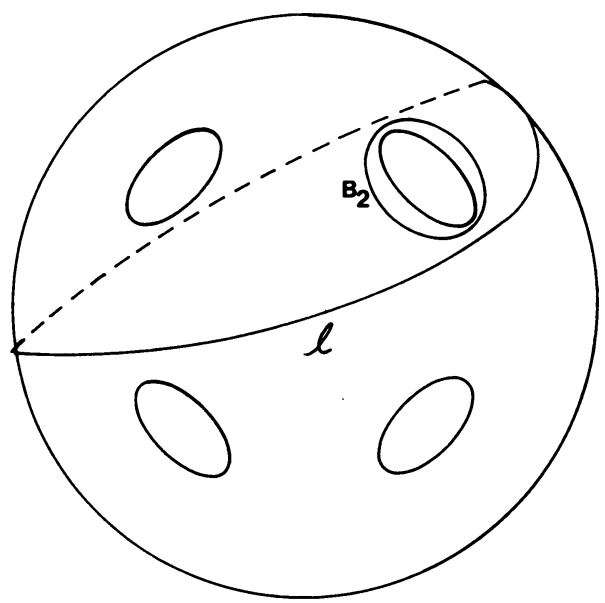

FIGURE 3

where $R$ is the $p \times p$ permutation matrix $\left(r_{i j}\right)$

$$
\begin{aligned}
& r_{i j}=\delta_{i, j+1}, \quad 1 \leq j \leq p-1, \\
& r_{i p}=\delta_{i, 1},
\end{aligned}
$$

Then $r_{p}^{-1} \circ g \circ r_{p}$ still acts as the identity on the homology of the surface, but the 2 in the last row is shifted one place to the right. Repeated conjugations shift the 2 to any position within the second vector of length $p$ in the bottom row. Compositions then generate all possible vectors in $\left(2 Z_{n}\right)^{p}$. Thus, we have induced any matrix of the form

$$
\left(\begin{array}{ccc}
I & 0 & 0 \\
0 & I & 0 \\
0 & v_{2} & 1
\end{array}\right),
$$

where $v_{2} \in\left(2 \mathbf{Z}_{n}\right)^{p}$.

It now remains to construct similar examples with any $v_{1} \in\left(2 Z_{n}\right)^{p}$ appearing in the first position of the bottom row. The idea, of course, is to interchange the roles of the $A$ curves and the $B$ curves, which can be accomplished either algebraically or geometrically.

To see this geometrically, we proceed with a construction analogous to the first part. Fix a simple closed curve $l$ representing $A_{1} B_{1} A_{1}^{-1} B_{1}^{-1} B_{2}^{-1}$, as in Figure 3 . The curves $B_{2}^{-1}$ and $l$ are homologous, and $f_{B_{2}}^{-1} \circ l$ induces the matrix

$$
\left(\begin{array}{ccc}
I & 0 & 0 \\
0 & I & 0 \\
w & 0 & 1
\end{array}\right) ;
$$

$w$ has $w_{2}=2$, all other entries zero. Then conjugation by the rotation map and compositions again generate all matrices

$$
\left(\begin{array}{ccc}
I & 0 & 0 \\
0 & I & 0 \\
v_{1} & 0 & 1
\end{array}\right)
$$

where $v_{1}$ is any element of $\left(2 \mathbf{Z}_{n}\right)^{p}$. 
Alternatively, because $\left(\begin{array}{cc}0 & -I \\ I & 0\end{array}\right)$ is a symplectic matrix, there is some $f \in \operatorname{Mod}_{p}$ so that $\rho(f)=\left(\begin{array}{cc}0 & -I \\ I & 0\end{array}\right)$. Then $\left(D f^{-1}\right)_{*}$ is of the form

$$
\left(\begin{array}{ccc}
0 & -I & 0 \\
I & 0 & 0 \\
w_{1} & w_{2} & 1
\end{array}\right) .
$$

Without trying to find $w_{1}$ and $w_{2}$, we can see that if $h \in \operatorname{Mod}_{p}$ (constructed above) induces

$$
\left(\begin{array}{lll}
I & 0 & 0 \\
0 & I & 0 \\
0 & v & 1
\end{array}\right),
$$

then $f^{-1} h f$ induces

$$
\left(\begin{array}{ccc}
I & 0 & 0 \\
0 & I & 0 \\
-v & 0 & 1
\end{array}\right) .
$$

Of course, compositions of these two kinds of examples give any $\left(v_{1}, v_{2}\right)=v \in$ $\left(2 \mathbf{Z}_{n}\right)^{2 p}$ as the bottom row.

REMARK. If $n$ is odd, and $v \in\left(\mathbf{Z}_{n}\right)^{2 p}$, and $I$ the $2 p \times 2 p$ identity matrix, then $\left[\begin{array}{ll}I & 0 \\ v & 1\end{array}\right]$ is induced by some $f \in \operatorname{Mod}_{p}$, for in this case $\left(2 Z_{n}\right)^{2 p}=\left(Z_{n}\right)^{2 p}$.

The sequence of Lemmas $2 \mathrm{a}-\mathrm{d}$ follows Earle's argument for the case $n=2$ quite closely [3, pp. 32-33].

LEMMA 2a. If $S$ is a symmetric $2 p \times 2 p$ matrix and $A$ is a $2 p \times 2 p$ matrix, both with coefficients in $\mathbf{Z}_{n}$, then

$$
(\operatorname{Diag} S) \cdot A-\operatorname{Diag}\left(A^{t} S A\right) \in\left(2 \mathbf{Z}_{n}\right)^{2 p} \text {. }
$$

REMARK. The statement essentially says that while (Diag $S) \cdot A \neq \operatorname{Diag}\left(A^{t} S A\right)$ in general, these are equal "mod 2." The proof is a direct calculation.

LEMMA 2b. Let $N$ be the $2 p \times 2 p$ matrix $\left(\begin{array}{ll}0 & I \\ 0 & 0\end{array}\right)$ and for $g \in \operatorname{Mod}_{p}$ define $\tau(g)=$ $\operatorname{Diag}\left(\rho_{n}\left(g^{-1}\right)^{t} N \rho_{n}\left(g^{-1}\right)\right)$. Then $\tau(g)$ satisfies the condition

$$
\tau(g h)-\tau(g)-\tau(h) \rho_{n}\left(g^{-1}\right) \in\left(2 \mathbf{Z}_{n}\right)^{2 p} .
$$

PROOF.

$$
\begin{aligned}
\tau(g h)- & \tau(g)-\tau(h) \rho_{n}\left(g^{-1}\right) \\
= & \operatorname{Diag}\left[\left(\rho_{n}(g h)^{-1}\right)^{t} N \rho_{n}(g h)^{-1}\right)-\operatorname{Diag}\left(\rho_{n}\left(g^{-1}\right)^{t} N \rho_{n}\left(g^{-1}\right)\right) \\
& -\operatorname{Diag}\left(\rho_{n}\left(h^{-1}\right)^{t} N \rho_{n}\left(h^{-1}\right)\right] \cdot \rho_{n}\left(g^{-1}\right) \\
= & \operatorname{Diag}\left[\rho_{n}\left(g^{-1}\right)^{t}\left[\rho_{n}\left(h^{-1}\right)^{t} N \rho_{n}\left(h^{-1}\right)-N\right] \rho_{n}\left(g^{-1}\right)\right] \\
& -\operatorname{Diag}\left[\rho_{n}\left(h^{-1}\right)^{t} N \rho_{n}\left(h^{-1}\right)\right] \cdot \rho_{n}\left(g^{-1}\right)
\end{aligned}
$$

This last expression is in $\left(2 \mathbf{Z}_{n}\right)^{2 p}$ by Lemma $2 \mathrm{a}$, using $A=\rho_{n}\left(g^{-1}\right)$ and $S=$ $\rho_{n}\left(h^{-1}\right)^{t} N \rho_{n}\left(h^{-1}\right)-N . S$ is symmetric here because $M^{t} N M-N$ is symmetric for a symplectic matrix $M$. 
LEMMA 2c. If $f \in \operatorname{Mod}_{p},\left(D f^{-1}\right)_{*}$ takes the matrix form

$$
\left(\begin{array}{cc}
\rho_{n}\left(f^{-1}\right) & 0 \\
\tilde{v}(f) & 1
\end{array}\right)
$$

This defines $\tilde{v}(f) \in\left(\mathbf{Z}_{n}\right)^{2 p}$ for $f \in \operatorname{Mod}_{p}$. Then $\tilde{v}$ satisfies the cocycle condition

$$
\tilde{v}(g h)=\tilde{v}(g)+\tilde{v}(h) \rho_{n}\left(g^{-1}\right)
$$

PROOF.

$$
\left(D g^{-1}\right)_{*}=\left(\begin{array}{cc}
\rho_{n}\left(g^{-1}\right) & 0 \\
\tilde{v}(g) & 1
\end{array}\right), \quad\left(D h^{-1}\right)_{*}=\left(\begin{array}{cc}
\rho_{n}\left(h^{-1}\right) & 0 \\
\tilde{v}(h) & 1
\end{array}\right)
$$

$g h$ acts by

$$
\left(D(g h)^{-1}\right)_{*}=\left(D h^{-1}\right)_{*}\left(D g^{-1}\right)_{*}=\left(\begin{array}{cc}
\rho_{n}\left(h^{-1} g^{-1}\right) & 0 \\
\tilde{v}(h) \rho_{n}\left(g^{-1}\right)+\tilde{v}(g) & 1
\end{array}\right)
$$

as claimed.

LEMMA 2d. For any $f \in \operatorname{Mod}_{p}, \tilde{v}(f)-\tau(f) \in\left(2 \mathbf{Z}_{n}\right)^{2 p}$.

PROOF. We first calculate $\tau$ and $\tilde{v}$ for the set $\left\{f_{A_{1}}, f_{B_{1}}, f_{C_{1}}, r_{p}\right\}$ of generators for $\operatorname{Mod}_{p}$. Denoting the $i$ th components of $\tau$ and $\tilde{v}$ by $\tau_{i}, \tilde{v}_{i}$, respectively, we find (interpreting the results from [9]) that

$$
\begin{aligned}
& \tau\left(r_{p}\right)=\text { zero vector, } \\
& \tau_{i}\left(f_{A_{1}}\right)= \begin{cases}0 & \text { if } i \neq p+1 \\
-1 & \text { if } i=p+1\end{cases} \\
& \tau_{i}\left(f_{B_{1}}\right)= \begin{cases}0 & \text { if } i \neq 1, \\
1 & \text { if } i=1,\end{cases} \\
& \tau_{i}\left(f_{C_{1}}\right)= \begin{cases}0 & \text { if } i \neq p+1 \text { or } p+2, \\
-1 & \text { if } i=p+1, \\
-1 & \text { if } i=p+2,\end{cases} \\
& \tilde{v}\left(r_{p}\right)=\text { zero vector, } \\
& \tilde{v}_{i}\left(f_{A_{1}}\right)= \begin{cases}0 & \text { if } i \neq p+1, \\
1 & \text { if } i=p+1,\end{cases} \\
& \tilde{v}_{i}\left(f_{B_{1}}\right)= \begin{cases}0 & \text { if } i \neq 1, \\
-1 & \text { if } i=1,\end{cases} \\
& \tilde{v}_{i}\left(f_{C_{1}}\right)= \begin{cases}0 & \text { if } i \neq p+1 \text { or } p+2, \\
-1 & \text { if } i=p+1, \\
1 & \text { if } i=p+2 .\end{cases}
\end{aligned}
$$

Notice that $\tau(g)-\tilde{v}(g) \in\left(2 \mathbf{Z}_{n}\right)^{2 p}$ for all four generators. Also, by the previous two lemmas, $\tau$ and $\tilde{v}$ satisfy the same cocycle condition, $\bmod \left(2 \mathbf{Z}_{n}\right)^{2 p}$. Thus, $\tau-\tilde{v} \in$ $\left(2 \mathbf{Z}_{n}\right)^{2 p}$ for any words in the generators, and therefore for all elements of $\operatorname{Mod}_{p}$.

PROOF OF THE PROPOSITION. If the matrix $\left[\begin{array}{cc}M & 0 \\ v & 1\end{array}\right]$ is induced by $f \in \operatorname{Mod}_{p}$, then $M=\rho_{n}\left(f^{-1}\right)$ is symplectic, $v=\tilde{v}(f)$ by definition, and Lemma $2 \mathrm{~d}$ says that condition $\operatorname{Diag}\left(M^{t} N M\right)-v \in\left(2 \mathbf{Z}_{n}\right)^{2 p}$ is satisfied. 
For the converse, we note that since the mapping $\rho_{n}: \operatorname{Mod}_{p} \rightarrow \operatorname{Sp}\left(p, \mathbf{Z}_{n}\right)$ is onto, there is some $h \in \operatorname{Mod}_{p}$ with $\rho_{n}\left(h^{-1}\right)=M$. Then $\operatorname{Diag}\left(M^{t} N M\right)-\tilde{v}\left(h^{-1}\right) \in$ $\left(2 \mathbf{Z}_{n}\right)^{2 p}$. By hypothesis, $\operatorname{Diag}\left(M^{t} N M\right)-v \in\left(2 \mathbf{Z}_{n}\right)^{2 p}$, so $v-\tilde{v}\left(h^{-1}\right) \in\left(2 \mathbf{Z}_{n}\right)^{2 p}$. Lemma 1 then says we can construct an element of $\operatorname{Mod}_{p}$ which induces $\left[\begin{array}{cc}I & 0 \\ v-\tilde{v}\left(h^{-1}\right) & 1\end{array}\right]$ and multiplying $h$ by this element gives a diffeomorphism in $\operatorname{Mod}_{p}$ which induces $\left[\begin{array}{cc}M & 0 \\ v & 1\end{array}\right]$.

PROOF OF THE COROLlARY. Recalling that under our identification we may speak of elements of $Q_{n}=\operatorname{Mod}_{p} / G_{p, n}$ as matrices, let $K$ be the subgroup of $\operatorname{Mod}_{p} / G_{p, n}$ consisting of elements of the form $\left[\begin{array}{ll}I & 0 \\ v & I\end{array}\right]$. Note that $K \approx\left(\mathbf{Z}_{n}\right)^{2 p}$ and $K$ is normal in $\operatorname{Mod}_{p} / G_{p, n}$. Let $H$ be the subgroup of elements of the form $\left[\begin{array}{cc}M & 0 \\ v & 1\end{array}\right]$. Then $H$ is isomorphic to $\operatorname{Sp}\left(p, \mathbf{Z}_{n}\right)$ and $H K$ is the entire group $Q_{n}$, so

$$
Q_{n}=K \ltimes H \approx\left(\mathbf{Z}_{n}\right)^{2 p} \ltimes \operatorname{Sp}\left(p, \mathbf{Z}_{n}\right) .
$$

Notice that a similar expression of the quotient as a semidirect product is not possible if $n$ is even. For a given symplectic matrix $M$, it is possible that $\left[\begin{array}{cc}M & 0 \\ 0 & 1\end{array}\right]$ is not in the quotient, for $\operatorname{Diag}\left(M^{t} N M\right) \notin\left(2 \mathbf{Z}_{n}\right)^{2 p}$. Thus, there is no apparent lifting of $\operatorname{Sp}\left(p, \mathbf{Z}_{n}\right)$ to $Q_{n}$.

\section{REFERENCES}

1. M. F. Atiyah, Riemann surfaces and spin structures, Ann. Sci. École Norm. Sup. (4) 4 (1971), 47-62.

2. C. J. Earle, Families of Riemann surfaces and Jacobi varieties, Ann. of Math. 107 (1978), 255-286.

3. _ Roots of the canonical divisor class over Teichmüller space, preprint.

4. D. Johnson, Spin structures and quadratic forms on surfaces, J. London Math. Soc. 22 (1980), 365-373.

5. W. B. R. Lickorish, A finite set of generators for the homeotopy group of a 2-manifold, Proc. Cambridge Philos. Soc. 60 (1964), 769-778.

6. D. Mumford, Abelian quotients of the Teichmüller modular group, J. Analyse Math. 18 (1967), 227-244.

7. C. L. Siegel, Topics in complex function theory, Vol. II, Wiley, New York, 1971.

8. P. L. Sipe, Roots of the canonical bundle of the universal Teichmüller curve, 'Thesis, Cornell Univ., 1979.

9. _ Roots of the canonical bundle of the universal Teichmüller curve and certain subgroups of the mapping class group, Math. Ann. 260 (1982), 67-92.

Department of Mathematics, Smith College, Northampton, Massachusetts 01063 (Current address)

Department of Mathematics, State University of NeW York at Stony BROOK, STONY BROOK, NEW YORK 11794-3651 